\title{
Potential for adrenergic imaging to serve as a unique tool for guidance of patient management during and after an acute ischemic event
}

\author{
Mark I. Travin, MD, FASNC \\ a Division of Nuclear Medicine, Department of Radiology, Montefiore Medical Center and the \\ Albert Einstein College of Medicine, Bronx, NY
}

Received Sep 8, 2016; accepted Sep 8, 2016

doi: $10.1007 / \mathrm{s} 12350-016-0675-0$

\section{See related article, pp. 572-580}

Since the first report in 1981 of adrenergic innervation imaging with the radiotracer iodine-123 metaiodobenzylguanidine $\left({ }^{123} \mathrm{I}-m \mathrm{IBG}\right)$, most cardiac work with it has focused on potential utility for assessing and managing patients who have heart failure with reduced ejection fraction ( $\mathrm{HFrEF}$ ) and associated lifethreatening ventricular arrhythmias. ${ }^{1}$ In particular, the ability of ${ }^{123} \mathrm{I}-m \mathrm{IBG}$ imaging to risk stratify $\mathrm{HFrEF}$ patients beyond what can be done with conventionally used clinical, ECG, and laboratory parameters promises to allow better guidance of invasive therapies such as implantable cardioverter defibrillators (ICDs) and left ventricular assist devices (LVAD), as well as assessment of the efficacy of pharmacologic therapies.

At the same time, much investigative work with ${ }^{123}$ I- $m$ IBG has also recognized its suitability for evaluating various other cardiac conditions, including ischemic heart disease. Adrenergic imaging in patients with coronary artery disease can provide unique insights because, in response to an ischemic insult, cardiac neurons behave differently from cardiac myocytes. Neurons have been shown to be more sensitive to oxygen deprivation than myocytes, ${ }^{2}$ and thus in the setting

Reprint requests: Mark I. Travin, MD, FASNC, Division of Nuclear Medicine, Department of Radiology, Montefiore Medical Center and the Albert Einstein College of Medicine, 111 E. 210th Street, Bronx, NY 10467-2490, USA; mtravin@attglobal.net

J Nucl Cardiol 2018;25:581-5.

$1071-3581 / \$ 34.00$

Copyright (C) 2016 American Society of Nuclear Cardiology. of myocardial ischemia ${ }^{123} \mathrm{I}-m \mathrm{IBG}$ defects can be present in the absence of perfusion tracer defects. ${ }^{3}$ As sympathetic axons travel basal to apical in superficial epicardial layers alongside coronary arterial pathways before penetrating into myocardium, the conducting fibers are exposed to damage from local ischemia along the pathway, and thus there may be ischemic sympathetic damage apical to areas of myocyte injury. ${ }^{4}$ Fallavollita and Canty have categorized such neuronal ischemic damage as dysinnervation defined as transient nerve dysfunction (also referred to as reversible neural stunning), or denervation defined as anatomic loss of fibers. ${ }^{5}$ Finally, it takes from 8 to 17 weeks for ischemic injured neurons to recover from an insult, with ${ }^{123} \mathrm{I}-$ $m$ IBG defects reported to be present months after resolution of the ischemic episode. ${ }^{6}$ Adrenergic image defects have been shown to persist longer than perfusion defects, ${ }^{7,8}$ and longer than defect on images using a fatty acid metabolic tracer such as iodine 123- $\beta$-Methyl- $p$ $\left[{ }^{123} \mathrm{I}\right]$-iodophenyl-pentadecanoic acid ( ${ }^{123} \mathrm{I}$-BMIPP). ${ }^{9}$

While ${ }^{123} \mathrm{I}-m \mathrm{IBG}$ defects in the setting of infarction or chronic ischemic hibernation have been shown to be associated with increased risk of arrhythmias ${ }^{7,8,10,11}$ and may have patient management utility in this regard, imaging with ${ }^{123} \mathrm{I}-m \mathrm{IBG}$ and related PET tracers has potential for assessing myocardial area at risk (AAR) in patients presenting with an acute coronary syndrome (ACS). In fact, use of radionuclide imaging to assess patients in this clinical setting, focusing on AAR and myocardial salvage after acute therapy, has been of interest since the advent of perfusion imaging. A 1981 report by Markis et al. of nine patients with acute MI showed that imaging after intracoronary injection of ${ }^{201} \mathrm{Tl}$ into the culprit artery before and again after intracoronary streptokinase could assess success of myocardial salvage as well as identify arterial 
reocclusion. ${ }^{12}$ Also in 1981, Maddahi reported on the use of pre-thrombolytic therapy intravenous ${ }^{201} \mathrm{Tl}$ imaging followed by post-therapy intracoronary ${ }^{201} \mathrm{Tl}$ imaging in five patients with acute MI, demonstrating the ability to determine the extent of myocardial salvage as well as the potential use of the technique to predict ventricular functional improvement, i.e., residual viability, with potential therefore to guide subsequent revascularization (at that time limited to surgery). ${ }^{13}$ Schofer et al. performed intracoronary injection of ${ }^{201} \mathrm{Tl}$ in 31 acute MI patients, 16 of whom also underwent simultaneous intracoronary injection of ${ }^{99 \mathrm{~m}} \mathrm{Tc}$-pyrophosphate (PYP) to assess local necrosis, and found a correlation between image evidence of myocardial salvage and improvement in ejection fraction. ${ }^{14}$

Of course, in the setting of an acute coronary event, use of ${ }^{201} \mathrm{Tl}$ that must be imaged immediately after injection is logistically difficult, especially in the current era of urgent intervention. The advent in the late 1980s of non-redistributing ${ }^{99 \mathrm{~m}} \mathrm{Tc}$-sestamibi facilitated protocols in which tracer injection could occur during the acute event but with imaging postponed for a few hours until after patient stabilization, with the images still depicting myocardial AAR at the time of injection. The acute images can then be compared with imaging performed days later to assess myocardial salvage. In a study of dogs undergoing MI inducing acute coronary occlusions, in which image findings could be correlated with postmortem tissue analysis of infarct size, Verani et al. reported that ${ }^{99 \mathrm{~m}} \mathrm{Tc}$-sestamibi imaging provided reliable demonstration of the presence of acute infarction, a good estimation of infarct size, and quantification of the extent of salvage myocardium after coronary reperfusion. ${ }^{15}$ Wackers et al., ${ }^{16}$ Gibbons et al., ${ }^{17}$ and Santoro et al., ${ }^{18}$ among others, demonstrated utility of this technique in people, with the latter study correlating image demonstrated myocardial salvage with ventricular functional recovery one month following the acute event.

From the onset, it was recognized that an advantage of a radionuclide image technique is that it depicts myocardial AAR differently from coronary anatomic methods. The latter generally involve sophisticated arithmetic and geometric algorithmic scoring methods based on vessel size and stenosis severity, but they do not account for the myriad of anatomic and physiologic factors, such as culprit stenosis shape and length, nonculprit distal stenoses and disease, vessel tone, collateral blood flow, and hemodynamic and metabolic variables, that determine AAR. Nuclear techniques integrate anatomy and physiology, and can show exactly how much myocardium is at risk at a given moment. In a study of 39 patients undergoing elective single-vessel coronary angiography who received intravenous ${ }^{99 \mathrm{~m}} \mathrm{Tc}-$ sestamibi immediately before balloon inflation and again 24 to 48 hours later, Haronian et al. ${ }^{19}$ found that nuclear perfusion image-derived AAR did not correlate well with that derived from anatomic algorithms. Christian et al. ${ }^{20}$ studied 89 patients receiving acute intervention (thrombolytic therapy or angioplasty) for acute MI, with imaging after ${ }^{99 \mathrm{~m}} \mathrm{Tc}$-sestamibi administration before reperfusion therapy and again about 1 week later, with a focus on using myocardium at risk and final infarct size to compare radionuclide with angiographic assessment of collateral flow. While there was a significant overall association between radionuclide and angiographic results, the correlation was held for anterior infarctions but not for smaller inferior infarctions possibly from the fact that in smaller infarctions collateral vessels may be less angiographically detectable.

More recently, radionuclide perfusion imagederived myocardial AAR has been used as a surrogate endpoint in at least 30 clinical acute MI trials. ${ }^{21}$ Perfusion imaging was able to validate $\mathrm{T} 2$-weighed cardiac magnetic resonance (CMR) assessment of $\mathrm{AAR},{ }^{22}$ and has served as a reference for assessing the validity of several anatomic angiographic score algorithms. ${ }^{23}$

As introduced above, using adrenergic tracers to assess AAR during an ACS can be done, with studies showing important differences in the response of neuronal tissues to ischemic injury from that of cardiomyocytes. In key study by Matsunari et al. ${ }^{24}$ involving 12 subjects without previous MI undergoing reperfusion therapy for an ACS, to assess AAR ${ }^{99 \mathrm{~m}} \mathrm{Tc}$ sestamibi was injected before revascularization with SPECT imaging performed 2 to 6 hours later. Infarct size was measured by a second resting ${ }^{99 \mathrm{~m}} \mathrm{Tc}$-sestamibi study 4 to 9 days later. Sympathetic neuronal damage was assessed with ${ }^{123} \mathrm{I}-m \mathrm{IBG}$ study performed 6 to 19 days after ACS onset. Figure 1 shows the typical pattern-infarct size based on post-MI imaging with perfusion tracers was significantly lower than AAR, while the post-MI ${ }^{123} \mathrm{I}-\mathrm{mIBG}$ images were nearly identical to the initial AAR. Similarly, a study from the same lab by Simões et al. ${ }^{8}$ of 67 post-MI patients imaged with ${ }^{123}$ I- $m$ IBG within 14 days of infarct onset, and studied with ${ }^{201} \mathrm{Tl} 2$ to 4 days later, showed the mean post-MI adrenergic defect size to be larger than the post-MI perfusion defect size in $90 \%$ of patients, with a mean mismatch size of $10 \% \pm 18 \%$ (range 0\%-59\%). Interestingly, the size of mismatch between adrenergic and perfusion images correlated with electrocardiographic (ECG) parameters-prolonged repolarization (i.e., higher heart rate-corrected QT interval) and evidence of delayed depolarization in the signal-averaged ECGconsidered to increase risk of ventricular arrhythmias, although in this small cohort event rates were too low to be significant. 


\section{Risk Area Infarct Size MIBG}

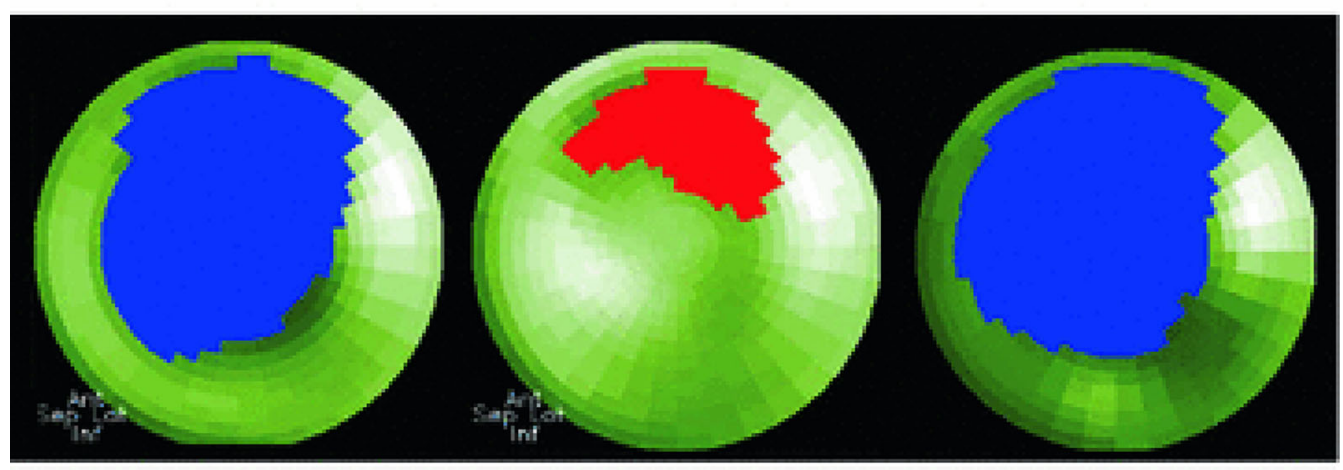

\section{$58 \%$ LV}
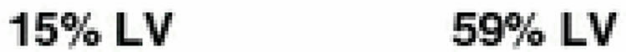

Figure 1. Extent of the MIBG defect correlates with area at risk during acute coronary occlusion. These polar tomograms were obtained from a patient with an acute anterior myocardial infarction. The risk area was quantified with ${ }^{99 \mathrm{~m}} \mathrm{Tc}$-sestamibi prior to reperfusion with percutaneous coronary intervention, and infarct size was documented from repeat imaging 1 week later. ${ }^{24}$ The defect in sympathetic nerve function assessed with MIBG was significantly larger than the area of infarction and was almost identical to the original extent of myocardial ischemia. Figure source Dr. Markus Schwaiger with permission. $L V$ left ventricle, $M I B G$ metaiodobenzylguanidine. From Fallavollita JA, Canty JM. Dysinnervated but viable myocardium in ischemic heart disease. J Nucl Cardiol 2010;17:1107-15,,$^{5}$ with permission of Springer.

Thus, adrenergic imaging with ${ }^{123} \mathrm{I}-m \mathrm{IBG}$ assesses an aspect of AAR different from perfusion tracers and therefore should provide unique insights into a patient's condition and future risk. From the few studies available it appears that adrenergic imaging can be performed conveniently several days after a revascularization intervention yet still depict how much myocardium was initially at risk. A question to be addressed is: in patients presenting with an ACS, how does ${ }^{123} \mathrm{I}-m \mathrm{IBG}$ imaging-determined AAR relate to anatomic techniques, and could adrenergic imaging be a more effective tool to assess myocardial salvage provided by cardioprotective interventions? To explore this matter, in this issue of the Journal of Nuclear Cardiology, Vauchot et al. $^{25}$ correlate ${ }^{123} \mathrm{I}-m \mathrm{IBG}$ image-determined AAR with that derived from the anatomic-modified APPROACH-score (ApAR) that is a semi-automatic quantification method based on the length and size of the branches of the culprit artery, validated in several studies ${ }^{26,27}$ and found to be useful for evaluating medical and interventional procedures to decrease infarct size. However, as described by Vauchot and colleagues, ApAR does not account for the many important anatomic and physiologic factors previously discussed, and does not provide models for branch vessel culprit lesions. The study protocol considered 96 patients without various clinical exclusion criteria who presented with acute ST-segment elevation myocardial infarction (STEMI) and were referred for primary PCI, but 20 patients with branch vessel culprit arteries and three others because of major collateral flow were excluded, and there were three refusals. In the final cohort of 70 patients who underwent ${ }^{123} \mathrm{I}$ $m$ IBG imaging on a cadmium-zinc-telluride (CZT) camera $13 \pm 7$ days after PCI, for the 50 patients with an occluded culprit artery, the ${ }^{123} \mathrm{I}-\mathrm{mIBG}$ area at risk (MAR) based on the summed dysinnervation score was highly correlated with the ApAR anatomic score, and also identified the same risk location regardless of how many other vessels had disease. However, for the 20 patients with a sub-occluded culprit artery there was no correlation with ApAR score that was most often higher than MAR (on average 4.5\%), although risk locations were the same. Thus, AAR derived from an ${ }^{123}$ I- $m$ IBG imaging study that was done up to about a month after the infarct intervention was well in accord with risk area derived from a validated anatomic model, but the nuclear imaging technique exposed inadequacies of this anatomic model that had to exclude 23 of 96 patients initially and likely overestimated AAR in an additional 20 patients with a suboccluded artery. The authors conclude that ${ }^{123} \mathrm{I}-\mathrm{mIBG}$ imaging often allows more accurate AAR assessment in patients whose arterial anatomic findings preclude applying standard algorithms, and could therefore potentially better test myocardial preservation techniques in acute MI patients whose culprit arteries have had some degree of spontaneous reperfusion. ${ }^{28}$ 
Of course, data from many more patients are needed to establish the validity of and practical clinical uses of ${ }^{123}$ I- $m$ IBG-derived AAR. Correlation with CMR AAR that some people consider a suitable standard, needs to be done. Also required will be further understanding of the performance and interpretation of adrenergic imaging on solid-state cameras and exploration of PET tracers, both of which improve tomographic adrenergic image quality that is frequently poor for ${ }^{123} \mathrm{I}-\mathrm{mIBG}$ acquired on standard gamma cameras, as well as definitive establishment of quantitative interpretation techniques that have important differences from those used for perfusion tracers. ${ }^{29}$

Nevertheless, the theoretic basis of adrenergic imaging in ACS is sound, the images provide information different from coronary anatomic algorithms and perfusion images, and the technique can be broadly and conveniently performed after an acutely ill patient has been stabilized. Further potential for using adrenergic imaging in this clinical setting can be seen when one goes beyond ${ }^{123} \mathrm{I}-m \mathrm{IBG}$ using PET tracers such as ${ }^{11} \mathrm{C}$ hydroxyephedrine, ${ }^{11} \mathrm{C}$-epinephrine, and ${ }^{11} \mathrm{C}$-phenylephrine that have been shown to depict different aspects of neuronal function, i.e., abnormalities of tracer uptake versus impairment of vesicular catecholamine storage in neurons in the infarct border zone. ${ }^{30}$ Such imaging should therefore provide new insights into infarct site healing, remodeling, and electrophysiologic stability. The promise of adrenergic nuclear imaging to uniquely assess pathobiology during and after an acute ischemic event can make it a powerful tool for helping to improve the outcome of these patients.

\section{References}

1. Travin MI. Clinical applications of myocardial innervation imaging. Cardiol Clin. 2016;34:133-47.

2. Dae MW, Herre JM, O'Connell JW, Botvinick EH, Newman D, Munoz L. Scintigraphic assessment of sympathetic innervation after transmural versus nontransmural myocardial infarction. J Am Coll Cardiol. 1991;17:1416-23.

3. Hartikainen J, Mustonen J, Kuikka J, Vanninen E, Kettunen R. Cardiac sympathetic denervation in patients with coronary artery disease without previous myocardial infarction. Am J Cardiol. 1997;80:273-7.

4. Kammerling JJ, Green FJ, Watanabe AM, Inoue H, Barber MJ, Henry DP, et al. Denervation supersensitivity of refractoriness in noninfarcted areas apical to transmural myocardial infarction. Circulation. 1987;76:383-93.

5. Fallavollita JA, Canty JM. Dysinnervated but viable myocardium in ischemic heart disease. J Nucl Cardiol. 2010;17:1107-15.

6. Inobe $\mathrm{Y}$, Kugiyama K, Miyagi H, Ohgushi M, Tomiguchi S, Takahashi M, Yasue H. Long-lasting abnormalities in cardiac sympathetic nervous system in patients with coronary spastic angina: quantitative analysis with iodine 123 metaiodobenzylguanidine myocardial scintigraphy. Am Heart J. 1997;134:112-8.
7. McGhie AI, Corbett JR, Akers MS, Kulkarni P, Sills MN, Kremers $\mathrm{M}$, et al. Regional cardiac adrenergic function using I-123 metaiodobenzylguanidine tomographic imaging after acute myocardial infarction. Am J Cardiol. 1991;67:236-42.

8. Simões MV, Barthel P, Matsunari I, Nekolla SG, Schömig A, Schwaiger M, et al. Presence of sympathetically denervated but viable myocardium and its electrophysiologic correlates after early revascularised, acute myocardial infarction. Eur Heart J. 2004;25:551-7

9. Watanabe K, Takahashi T, Miyajima S, Hirokawa Y, Tanabe N, Kato $\mathrm{K}$, et al. Myocardial sympathetic denervation, fatty acid metabolism, and left ventricular wall motion in vasospastic angina. J Nucl Med. 2002;43:1476-81.

10. Stanton MS, Tuli MM, Radtke NL, Heger JJ, Miles WM, Mock $\mathrm{BH}$, et al. Regional sympathetic denervation after myocardial infarction in humans detected noninvasively using I-123metaiodobenzylguanidine. J Am Coll Cardiol. 1989;14:1519-26.

11. Fallavollita JA, Heavey BM, Luisi AJ Jr, Michalek SM, Baldwa S, Mashtare TL, et al. Regional myocardial sympathetic denervation predicts the risk of sudden cardiac arrest in ischemic cardiomyopathy. J Am Coll Cardiol. 2014;63:141-9.

12. Markis JE, Malagold M, Parker JA, Silverman KJ, Barry WH, Als $\mathrm{AV}$, et al. Myocardial salvage after intracoronary thrombolysis with streptokinase in acute myocaridal infarction. Assessment by intracoronary thallium-201. N Engl J Med. 1981;305:777-82.

13. Maddahi J, Ganz W, Ninomiya K, Hashida J, Fishbein MC, Mondkar A, et al. Myocardial salvage by intracoronary thrombolysis in evolving acute myocardial infarction: Evaluation using intracoronary injection of thallium-201. Am Heart J. 1981;102:664-74.

14. Schofer J, Mathey DG, Montz R, Bleifeld W, Stritzke P. Use of dual intracoronary scintigraphy with thallium-201 and technetium$99 \mathrm{~m}$ pyrophosphate to predict improvement in left ventricular wall motion immediately after intracoronary thrombolysis in acute myocardial infarction. J Am Coll Cardiol. 1983;2:737-44.

15. Verani MS, Jeroudi MO, Mahmarian JJ, Boyce TM, Borges-Neto S, Patel B, Bolli R. Quantification of myocardial infarction during coronary occlusion and myocardial salvage after reperfusion using cardiac imaging with technetium-99m hexakis 2-methoxyisobutyl isonitrile. J Am Coll Cardiol. 1988;12:1573-81.

16. Wackers FJT, Gibbons RJ, Verani MS, Kayden DS, Pellikka PA, Verani MS, et al. Serial quantitative planar technetium-99m isonitrile imaging in acute myocardial infarction: Efficacy for noninvasive assessment of thrombolytic therapy. J Am Coll Cardiol. 1989;14:861-73.

17. Gibbons RJ, Verani MS, Behrenbeck T, Pellikka PA, O'Connor MK, Mahmarian JJ, et al. Feasibility of tomographic ${ }^{99 \mathrm{~m}} \mathrm{Tc}-\mathrm{Hex}-$ akis-Isonitrile imaging for the assessment of myocardial area at risk and the effect of treatment in acute myocardial infarction. Circulation. 1989;80:1277-86.

18. Santoro GM, Bisi G, Sciagrà R, Leoncini M, Fazzini PF, Meldolesi U. Single photon emission computed tomography with technetium-99m hexakis 2-methoxyisobutyl isonitrile in acute myocardial infarction before and after thrombolytic treatment: Assessment of salvaged myocardium and prediction of late functional recovery. J Am Coll Cardiol. 1990;15:301-14.

19. Haronian HL, Remetz MS, Sinusas AJ, Baron JM, Miller HI, Cleman MW, et al. Myocardial risk area defined by tehcnetium$99 \mathrm{~m}$ sestamibi imaging during percutaneous transluminal coronary angioplasty: Comparison with coronary angiography. J Am Coll Cardiol. 1993;22:1033-43

20. Christian TF, Schwartz RS, Gibbons RJ. Determinants of infarct size in reperfusion therapy for acute myocardial infarciton. Circulation. 1992;86:81-90. 
21. Miller TD, Sciagrà R, Gibbons RJ. Application of tehcnetium-99m sestamibi single photon emission computed tomography in acute myocardial infarction: Measuring the efficacy of therapy. Q J Nucl Med Mol Imaging. 2010;54:213-29.

22. Carlsson M, Ubachs JF, Hedstrom F, Heiberg E, Jovinge S, Arheden H. Myocardium at risk after acute infarction in humans on cardiac magnetic resonance: quantitative assessment during follow-up and validation with single-photon emission computed tomography. J Am Coll Cardiovasc Imging. 2009;2:569-76.

23. Rodriguez-Palomares JF, Alonso A, Marti G, Aguadé-Bruix S, Gonzalez-Alujas MT, Romero-Farina G, et al. Quantification of myocardial area at risk in the absence of collateral flow: The validation of angiographic scores by myocardial perfusion singlephoton emission computed tomography. J Nucl Cardiol. 2013;20:99-110.

24. Matsunari I, Schricke U, Bengel FM, Haase H, Barthel P, Schmidt $\mathrm{G}$, et al. Extent of cardiac sympathetic neuronal damage is determined by the area of ischemia in patients with acute coronary syndrome. Circulation. 2000;101:2579-85.

25. Vauchot F, Bouallegue B, Hedon C, Piot C, Roubille F, MarianoGoulart D. Assessment of the area at risk after acute myocardial infarction: interests of 123I-MIBG SPECT compared with the angiographic APPROACH-score. J Nucl Cardiol. 2016. doi: 10.1007/s12350-016-0644-7.

26. Ortiz-Pérez JT, Meyers SN, Lee DC, Kansal P, Klocke FJ, Holly TA, et al. Angiographic estimates of myocardium at risk during acute myocardial infarction: Validation study using cardiac magnetic reaonance imaging. Eur Heart J. 2007;28:1750-8.

27. Graham MM, Faris PD, Ghali WA, Galbraith PD, Norris CM, Badry JT, et al. Validation of three myocardial jeopardy scores in a population-based cardiac catheterization cohort. Am Heart J. 2001;142:254-61.

28. Roubille F, Mewton N, Elbaz M, Roth O, Prunier F, Cung TT, et al. No post-conditioning in the human heart with thrombolysis in myocardial infarction flow 2-3 admission. Eur Heart J. 2014;35:1675-82.

29. Travin MI. It's not all in the numbers. J Nucl Cardiol. 2016;23:436-41.

30. Lautamaki R, Sasano T, Higuchi T, Nekolla SG, Lardo A, Holt $\mathrm{DP}$, et al. Multiparametric molecular imaging provides mechanistic insights into sympathetic innervation impairment in the viable infarct border zone. J Nucl Med. 2015;56:457-63. 